\title{
How Does Individual Recognition Evolve? Comparing Responses to Identity Information in Polistes Species with and Without Individual Recognition
}

\author{
Michael J. Sheehan, Michael A. Straub \& Elizabeth A. Tibbetts \\ Ecology and Evolutionary Biology, University of Michigan, Ann Arbor, MI, USA
}

\section{Correspondence}

Michael J. Sheehan, Ecology and Evolutionary

Biology, University of Michigan, Ann Arbor,

MI 48109, USA.

E-mail:msheehan@berkeley.edu

Received: May 9, 2013

Initial acceptance: June 18, 2013

Final acceptance: October 31, 2013

(L. Ebensperger)

doi: 10.1111/eth.12191

Keywords: individual recognition; social brain hypothesis; sensory drive; sensory drive; signal evolution; comparative cognition

\begin{abstract}
A wide range of complex social behaviors are facilitated by the recognition of individual conspecifics. Individual recognition requires sufficient phenotypic variation to provide identity information as well as receivers that process and respond to identity information. Understanding how a complex trait such as individual recognition evolves requires that we consider how each component has evolved. Previous comparative studies have examined phenotypic variability in senders and receiver learning abilities, although little work has compared receiver responses to identity information among related species with and without individual recognition. Here, we compare responses to identity information in two Polistes paper wasps: $P$. fuscatus, which visually recognizes individuals, and P. metricus, which does not normally show evidence of individual recognition. Although the species differ in individual recognition, the results of this study show that receiver responses to experimentally manipulated identity information are surprisingly similar in both species. Receivers direct less aggression toward identifiable individuals than unidentifiable individuals. Therefore, the responses necessary for individual recognition may pre-date its evolution in the P. fuscatus lineage. Additionally, our data demonstrate the apparent binary differences in a complex behavior between the two species, such as individual recognition, likely involve incremental differences along a number of axes.
\end{abstract}

\section{Introduction}

The ability to recognize and remember previous interactions with particular individuals is a key component of a wide range of complex social behaviors. Individual recognition is essential to the individuated relationships found in many vertebrate and some invertebrate societies (Bshary et al. 2002; Cheney 8 Seyfarth 2007; Dunbar \& Shultz 2007; Gherardi et al. 2012) and has been theorized to influence cooperation (Crowley et al. 1996; Stevens et al. 2005; Brosnan et al. 2010), dominance hierarchies (Barnard \& Burk 1979), and territorial acquisition and maintenance (Jaeger 1981; Stamps 1987).

Empirical research on the distribution of individual recognition among animals has largely focused on two main areas. First, many studies have experimentally demonstrated the presence of recognition abilities across taxa and contexts (Beer 1969; Bee \& Gerhardt 2002; Tibbetts 2002; Torriani et al. 2006; Carazo et al. 2008). Second, other studies have examined the extent of individual knowledge and memory (Godard 1991; Bergman et al. 2003; Gherardi \& Atema 2005; Sheehan \& Tibbetts 2008). The distribution of individual recognition across taxa is often described as being limited by species' cognitive abilities (Wilson 1975; Lehmann \& Perrin 2002; Doorn et al. 2003; Stevens et al. 2005), although little research has in fact empirically examined which factors limit the distribution of individual recognition in different taxa.

To be functional, individual recognition requires that a species possess three characteristics (Sherman 
et al. 1997): (1) variable phenotypes of senders that provide identity information, (2) receivers that can distinguish among individuals, and (3) receivers that respond differentially to individuals based on their identity and history of past interactions. Differences in individual recognition between species may arise from differences in any of these three characteristics: sender phenotypes, receiver processing, and/or receiver responses. Understanding which traits involved in recognition, if any, are shared with relatives that lack individual recognition is an important first step in elucidating the processes through which complex social behaviors utilizing individual recognition evolve.

Variation in sender phenotypes may be widespread, even in species that lack established individual recognition. A number of species lacking individual recognition possess traits that are variable among individuals but consistent within an individual (McCulloch et al. 1999; Cure et al. 2009; Dreier \& D'Ettorre 2009), suggesting that identity information may not limit the initial evolution of individual recognition in some species.

Receiver processing abilities are also unlikely to limit the evolution of individual recognition. Animals are generally adept at pattern recognition (e.g. Avargues-Weber et al. 2011). Honeybees are a prime example of this, as they can be trained to differentiate among human faces although there is no reason to believe that bees typically recognize individual humans or that bees have evolved specialized learning mechanisms for distinguishing among human faces (Dyer et al. 2005). Instead, bees likely learn human face images in the same way that they learn flower images. Operant conditioning studies have also shown that species that lack individual recognition are nevertheless able to distinguish among conspecifics when tasked with doing so and provided sufficient identity information (Loesche et al. 1991; Sheehan \& Tibbetts 2011). Although both identity information and receiver processing may evolve to facilitate more efficient recognition, pre-existing variation in sender phenotypes and adequate receiver processing abilities may be widespread.

In contrast to the evolution of identity signatures and receiver processing, the evolution of receiver responses to identity information remains poorly understood. Receiver responses are likely to be the selective force driving the evolution of recognition behavior because they reflect the action component of recognition (Liebert \& Starks 2004). Receivers respond to identity information in a variety of ways depending on the particular context (e.g. changes in aggression in territorial neighbors, the preferential feeding and care of a parent's chicks) although responses can be classified into three broad categories based on their average effects on sender fitness-beneficial, neutral, and harmful. The effects of receiver responses on senders are critical for predicting the evolution of individual recognition, as different responses will have different consequences for the evolution of identity information (Beecher 1988). Where receiver responses to identity information are beneficial for senders, the evolution of identity signals is favored (Dale et al. 2001; Sheehan \& Tibbetts 2009). In contrast, harmful receiver responses should favor concealment of individual identity (Johnstone 1997), which may prevent the evolution of individual recognition.

Species comparisons provide a powerful method for testing the factors that facilitate the evolution of individual recognition. In particular, do appropriate receiver responses pre-date established individual recognition or are responses the result of selection for individual recognition? Recognition requires the interaction of sender phenotypes and receiver behavior (Sherman et al. 1997). The initial evolution of recognition, then, would be facilitated by pre-existing variation in sender phenotypes or appropriate receiver behavior (Scott-Phillips et al. 2012). If two species differ in the occurrence of established individual recognition, but have similar responses to identity information, the differences in individual recognition behavior must be due to factors other than receiver responses. For example, there may not be sufficient naturally occurring variation to distinguish individuals. Comparing receiver responses to identity information among closely related species will shed light on the extent to which appropriate receiver responses may either pre-date or be the result of selection for individual recognition.

Here, we experimentally examine receiver responses to identity information in Polistes metricus, a paper wasp that lacks individual recognition (Sheehan \& Tibbetts 2010). We compare our results with $P$. metricus to previously published results from a closely related wasp, P. fuscatus (Sheehan \& Tibbetts 2009). Polistes fuscatus wasps possess strikingly variable color patterns on their face and abdomen that are used for individual recognition (Fig. la, Tibbetts 2002). Our previous work has demonstrated that the variable color patterns in $P$. fuscatus are identity signals that have evolved as a result of selection for recognizability (Sheehan \& Tibbetts 2009, 2010). Nest founding queens benefit by receiving less aggression when they are easily identifiable (Sheehan \& Tibbetts 2009). Additionally, P. fuscatus show evidence of specialized 
(a)

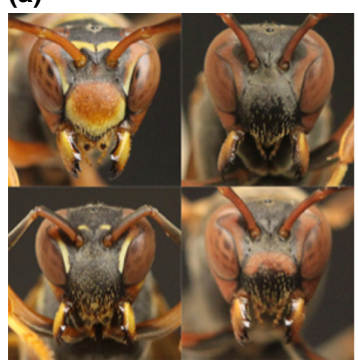

(b)

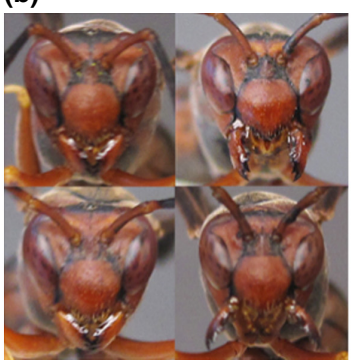

(c)

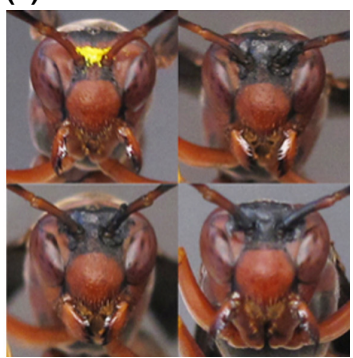

Fig. 1: (a) Polistes fuscatus wasps have distinctive, identity-signaling color patterns used for individual recognition (Tibbetts 2002; Sheehan \& Tibbetts 2008). (b) Polistes metricus wasps lack variable color patterns and do not recognize individuals (Sheehan \& Tibbetts 2010). (c) Example of the experimental manipulation of Polistes metricus wasps. Each experimental group contained four wasps collected at different sites. The appearances of all wasps in the group were altered with either yellow or black enamel paint. In each group, three individuals were given the same appearance (i.e. common phenotypes), while one was given a unique appearance (i.e. an identifiable phenotype). The color of the unique wasp was balanced across groups, so that the unique wasp was painted yellow in half the groups and black in half the groups.

face-specific mechanisms for processing identity information (Sheehan $\&$ Tibbetts 2011). Polistes metricus is closely related to $P$. fuscatus (Fig. 2, Pickett $\&$ Carpenter 2010; Buck et al. 2012), but lacks color pattern variation (Fig. lb) and shows no evidence of individual recognition (Sheehan \& Tibbetts 2010). Although $P$. metricus has difficulty differentiating between images of conspecifics, P. metricus can differentiate between images of $P$. fuscatus in an operant training paradigm (Sheehan \& Tibbetts 2011). Therefore,
P. metricus are capable of distinguishing between wasps if there is sufficient identity information. Previous work has not tested whether P. metricus receivers respond to identity information during social interactions.

We test receiver responses to identity information in $P$. metricus by assessing how experimentally increasing identity information influences receiver behavior. Distinctive phenotypes allow individuals to discriminate among potential social partners and

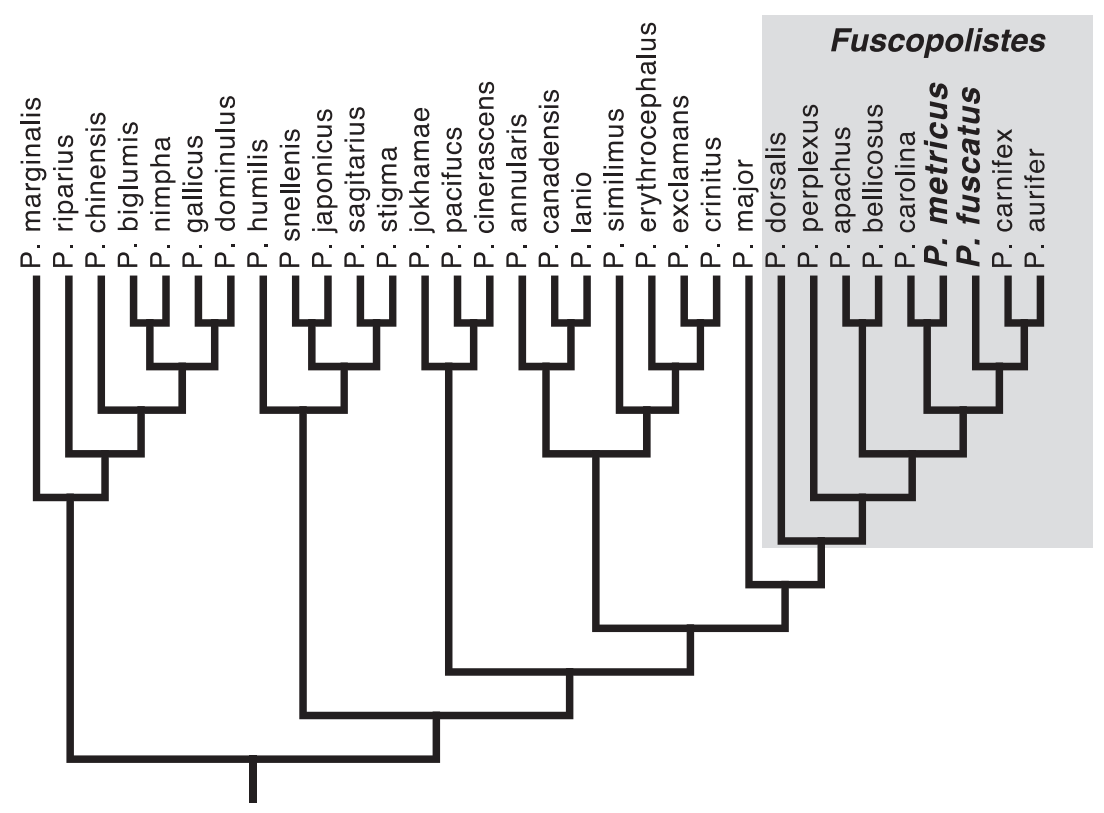

Fig. 2: Cladogram of Polistes highlighting the subgenus Fuscopolistes, which contains both $P$. fuscatus and $P$. metricus. The tree is drawn from data presented by Pickett \& Carpenter (2010) based on a phylogeny of the Vespid wasps using both morphological and molecular characters. Please note that in this cladogram, branch lengths are not scaled for relative divergence as such a phylogeny is currently unavailable for the genus. However, recent attempts to delineate species within the Fuscopolistes subgenus in the eastern USA (where both $P$. fuscatus and $P$. metricus are found) using DNA barcodes found extremely low levels of divergence among species suggesting a relatively recent radiation within this group (Buck et al. 2012). 
associate information with their identity, such as the outcome of a past interaction. Previous work in a closely related species with established individual recognition, P. fuscatus, has shown that distinctive, identifiable individuals receive less aggression than individuals with common, unrecognizable appearances even among groups of previously unfamiliar individuals (Sheehan \& Tibbetts 2009). Indeed, lower aggression toward familiar individuals is the hallmark of individual recognition in paper wasps (Tibbetts 2002; Sheehan \& Tibbetts 2008) suggesting that reduced aggression toward distinctive unfamiliar individuals is the result of learning individual identity during the course of the trials. Therefore, we experimentally increase identity information in P. metricus to test the following question: does identity information influence receiver behavior and if so, what is the likely fitness consequence of the response for senders?

There are three possible outcomes that might emerge from our experiment. First, identity information may have no influence on $P$. metricus receiver behavior, suggesting that variation in receiver responses may contribute to differences in recognition between the two species. Second, $P$. metricus receivers may respond to identity information in a way that harms senders, such as increasing the rate of aggression toward identifiable individuals, suggesting that P. metricus receiver behavior could favor the lack of identity information in senders and hinder the evolution of individual recognition. Third, P. metricus receivers might respond to identity information by reducing aggression toward recognizable individuals with whom they have interacted. Such a response would be expected to favor the evolution of identity signals (Dale et al. 2001; Sheehan \& Tibbetts 2009), potentially facilitating the evolution of individual recognition under the appropriate contexts. Additionally, this outcome would suggest that $P$. metricus receivers were likely associating social information with identifying phenotypes.

\section{Methods}

Polistes metricus is a common paper wasp found throughout the eastern United States that is closely related to P.fuscatus (Fig. 2, Pickett \& Carpenter 2010). As with all temperate paper wasps, P. metricus colonies are initiated each spring by foundresses that have recently emerged from diapause. We used foundresses collected from human structures in three locations during April and May of 2007: Ann Arbor, Michigan $\left(43^{\circ} 16^{\prime} \mathrm{N}, 83^{\circ} 44^{\prime} \mathrm{W}\right)$, Columbus, Ohio $\left(39^{\circ} 59^{\prime} \mathrm{N}, 82^{\circ} 59^{\prime} \mathrm{W}\right)$ and Knoxville, Tennessee $\left(35^{\circ} 58^{\prime}\right.$
$\left.\mathrm{N}, 8^{\circ} 56^{\prime} \mathrm{W}\right)$. All nests were collected in the early founding phase when nests were small and had eggs or early instar larvae. They were brought into the laboratory, housed individually and provided ad libitum access to sugar cubes and water.

Using 80 foundresses, we created 20 different groups of four, unfamiliar weight-matched individuals collected from locations at least $1 \mathrm{~km}$ apart to reduce the possibility individuals had not previously interacted as wasps tend to be highly philopatric and dispersal distances of greater than $300 \mathrm{~m}$ have not been observed (Klahn 1979; Hirose \& Yamasaki 1984; Makino et al. 1987). All groups contained foundresses from at least two different collection regions. We experimentally altered the appearance of each wasp, so there were three foundresses with a similar appearance and one foundress with a unique appearance in each group. This treatment is meant to mimic a situation where a rare mutation arises causing an increase in identity information that could be used for discrimination or recognition. For all foundresses in each trial, we painted the region just above the antenna with either black or yellow paint (Fig. 1c). Previous studies have shown that, while $P$. metricus color patterns are largely invariant, there is slight variation in this region of the face (Sheehan $\&$ Tibbetts 2010). This region, then, is a plausible candidate for the location that a proto-identity signal may evolve in this lineage. Our paint treatments provided variation in this region that is roughly similar to that seen in P. fuscatus in the same region (Fig. 1a), although beyond what is naturally found in P. metricus populations (Fig. lb). The distribution of color patterns was balanced across trials such that the identifiable wasp was yellow in half the trials and black in half the trials. Identifiable wasps were chosen randomly from among the four possible individuals. To allow individual identification by the experimenters, each wasp was given two small dots of red paint on the top of their thorax in a unique pattern. The red dots are unlikely to increase the perception of identity information as they are small and on the backs. Additionally, data from other hymenopterans suggest that Polistes wasps are unlikely to see red (Briscoe \& Chittka 2001). All groups contained sizedmatched foundresses (mean foundresses size: $0.177 \pm 0.003 \mathrm{~g}$, range of mean foundress sizes across trials: $0.143-0.222 \mathrm{~g}$, mean coefficient of variation of foundress size: $0.04 \pm 0.007)$. There were not consistent differences in size between foundresses based on their color treatment $\left(F_{1,74}=1.98, \mathrm{p}=0.16, \mathrm{n}=76\right)$ or whether or not they received an identifiable marking $\left(F_{1,74}=0.16, \mathrm{p}=0.69, \mathrm{n}=76\right)$. Trials were conducted from June 12 to 13, 2007. 
After the paint treatments dried, the wasps were placed in a small container $(8 \times 8 \times 2 \mathrm{~cm})$ and their interactions were filmed for $2 \mathrm{~h}$. During the early nest founding period, foundresses typically compete with numerous rivals over dominance rank in areas without nests or resources (Roeseler 1991; MJS and EAT personal observations). Our experimental setup mimics this situation, so foundresses readily engage in aggressive competition in the trials. The tapes were scored for aggressive acts such as darts, lunges, bites, grapples and mounts - all of which are stereotyped behaviors commonly used to assess wasp aggression (West Eberhard 1969; Reeve \& Nonacs 1992; Strassmann et al. 2004). Straub scored the videotapes blind to the treatments, experimental design, and predictions of the experiment. One tape was damaged and unable to be viewed, so the sample size was reduced to 19 from 20 trials.

We analyzed whether the levels of aggression initiated and received differed between identifiable and indistinguishable wasps. Aggression was markedly elevated in the initial part of the trial in $P$. metricus (Fig. S1, lst $\mathrm{h}-280.21 \pm 74.60$ aggressive acts, $2 \mathrm{nd} \mathrm{h}$ $-176.32 \pm 41.91 ; \mathrm{N}=19$ trials, paired $t$-test, $t_{18}=2.9$, $\mathrm{p}=0.0095$ ), so we measured the effect of distinctiveness during the first and second half of the trials separately. To account for differences in overall levels of aggression across trials $(\widetilde{x}=261$ aggressive acts, 25 th percentile $=157.5$ aggressive acts, 75 th percentile $=531.5$ aggressive acts), we calculated a standardized score for each wasp. To do so, we subtracted the mean number of aggressive acts initiated or received in a trial from the number of aggressive acts initiated or received by the focal wasp. This score was divided by the standard deviation of aggression within a given trial. With this technique, the standard aggression scores of distinctive wasps could be compared to the overall trial averages (set to zero) with one sample t-tests (Sokal \& Rohlf 1995). Positive standardized scores indicate that the wasp received more aggression than others in her trial, while negative standardized scores indicate that the wasp received less aggression than others in her trial. We conducted the same analyses of the data from our previous experiment with P. fuscatus (Sheehan \& Tibbetts 2009). The earlier experiment followed the same procedures, except that we painted clypei either black or yellow in P. fuscatus. Standard scores are reported as the mean number of standard deviations that identifiable wasp aggression (given or received) differed from the trial average. In addition, we report the standard error around the mean standard score. Variation reported around means is reported as standard error throughout. All tests described are two-tailed.

\section{Results}

During the 1 st h of the $P$. metricus trials, when aggression was most intense (see Fig. S1, Methods), identifiable wasps received less aggression than non-identifiable wasps (Fig. 3, mean aggressive acts received $=0.33 \mathrm{SD}$ less than the trial average \pm $\left.0.15 \mathrm{SE}, \mathrm{N}=19, t_{18}=-2.14, \mathrm{p}<0.05\right)$. Identifiable wasps were equally aggressive as the other wasps (mean aggressive acts initiated $=0.013$ SD less than the trial average $\pm 0.17 \mathrm{SE}, \mathrm{N}=19, t_{18}=-0.08, \mathrm{p}=$ 0.94), so the lower levels of aggression received are not a by-product of the identifiable wasps being less aggressive. The color a wasp was painted did not affect the amount of aggression it received during this period (black $=0.03 \mathrm{SD}$ more that than the trial average $\pm 0.14 \mathrm{SE}, \mathrm{N}=39, t_{38}=0.23, \mathrm{p}=0.82$; yellow $=$ $0.03 \mathrm{SD}$ less that than the trial average $\pm 0.14 \mathrm{SE}$, $\left.\mathrm{N}=37, t_{36}=-0.24, \mathrm{p}=0.81\right)$. Additionally, the aggression received by the identifiable foundress was not influenced by the mean mass of foundresses in her trial (linear regression: $F_{1,17}=0.20, r^{2}=-0.05$, $\mathrm{p}=0.66, \mathrm{n}=19$ identifiable wasps) or the standardized mass of the identifiable foundress relative to those in her trial (linear regression: $F_{1,17}=1.24$, $r^{2}=0.013, \mathrm{p}=0.28, \mathrm{n}=19$ identifiable wasps).

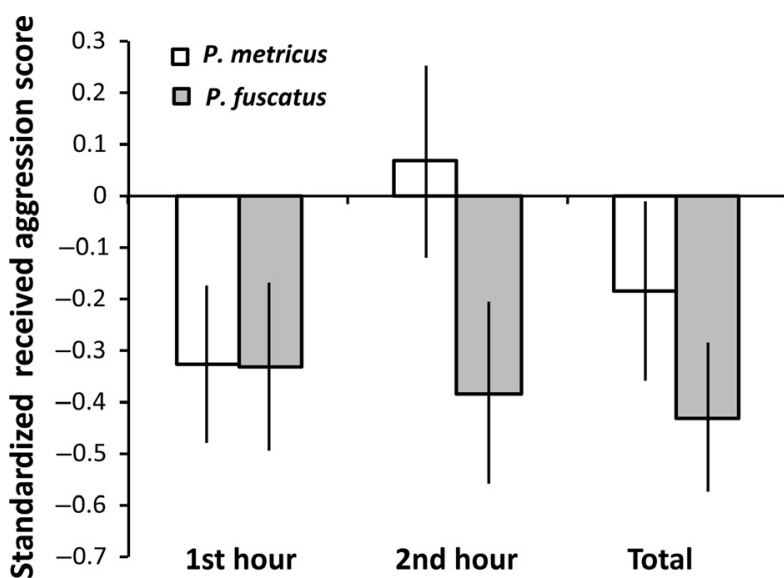

Fig. 3: Mean standardized aggression scores ( \pm SEM) for the wasps with distinctive, identifiable phenotypes. Individuals with phenotypes experimentally manipulated to provided identity information receive less aggression than the average individual within the trial. Standardized aggression scores show how much aggression an individual received as the number of standard deviations from the mean of the trial in which they participated. Negative values indicate that identifiable individuals received less aggression than the trial mean. Reduced aggression toward identifiable individuals is only detectable in the $1 \mathrm{st} \mathrm{h}$ of the trials in Polistes metricus, although the effect is consistent in Polistes fuscatus. See text for statistics. 
Therefore, the presence of experimentally added identity information per se rather than a particular color or size of the foundress was responsible for the reduction in aggression.

In the $2 \mathrm{nd} \mathrm{h}$ of the trials, when aggression levels were significantly lower (see Fig. S1, Methods), there was no discernable effect of identity information on the distribution of aggression among wasps (Fig. 3, mean aggressive acts received $=0.067 \mathrm{SD}$ more than the trial average $\pm 0.19 \mathrm{SE}, \mathrm{N}=19, t_{18}=0.36$, $p=0.73)$. Consistent with the reduced effect of identify information in the $2 \mathrm{nd} h$, the benefit of identity information is obscured if the entire 2 -h trial is analyzed (Fig. 3, mean aggressive acts received $=0.18 \mathrm{SD}$ less than the trial average $\pm 0.17 \mathrm{SE}, \mathrm{N}=19, t_{18}=$ $-1.06, \mathrm{p}=0.3$ ).

The responses to identity information at the beginning of the P. metricus trials mirror those observed in the same experiment performed with P. fuscatus (Fig. 3, mean aggressive acts received $=0.33$ SD less than the trial average $\pm 0.16 \mathrm{SE}, \mathrm{N}=18, t_{17}=-2.02$, $\mathrm{p}=0.06$ ). Unlike, in P. metricus, however, levels of aggression did not decline over time in P. fuscatus $(1 \mathrm{st} \mathrm{h}=116.11+10.31$ aggressive acts; $2 \mathrm{nd} \mathrm{h}=$ $138.33+44.45$ aggressive acts; $\mathrm{N}=18, t_{18}=-0.51$, $\mathrm{p}=0.61)$ and the response to identity information was increasingly detectable in the $2 \mathrm{nd} h$ (mean aggressive acts received $=0.38 \mathrm{SD}$ less than the trial average $\pm 0.18 \mathrm{SE}, \mathrm{N}=18, t_{17}=2.15, \mathrm{p}<0.05$ ) or when analyzing both hours together (mean aggressive acts received $=0.43 \mathrm{SD}$ less than the trial average $\pm 0.15 \mathrm{SE}, \mathrm{N}=18, t_{17}=-2.95, \mathrm{p}=0.009$ ).

\section{Discussion}

When provided with sufficient identity information, $P$. metricus receivers discriminate between individuals. Foundresses direct less aggression toward identifiable individuals than unidentifiable individuals in the course of staged social interactions. This pattern of behavior is similar to what we previously reported using the same experimental paradigm in P. fuscatus (Sheehan \& Tibbetts 2009), a close relative that recognizes individuals using variable color patterns (Tibbetts 2002). There were also some differences in behavior toward identifiable individuals across species. Specifically, a significant difference in aggression toward identifiable and indistinguishable individuals was only detectable in the $1 \mathrm{st} \mathrm{h}$ of the trials in P. metricus, which coincided with a period of elevated aggression within the groups (Fig. S1). An examination of patterns of aggression across the trials shows that identifiable wasps tend to receive less aggression than indistinguishable wasps during the peak of aggression at the outset of the trial (Fig. S1). Levels of aggression decline in both groups of wasps, although levels of aggression toward identifiable individuals bottom out more rapidly than in indistinguishable individuals (Fig. S1). In P. fuscatus, identifiable individuals received less aggression than indistinguishable individuals over all time periods with no observed decline in aggression. This suggests that the effect of identity information on receiver behavior, while detectable, is weaker in P. metricus compared to $P$. fuscatus and most apparent when during periods of intense aggression. The overall reduction in aggression in P. metricus that is absent in P. fuscatus also suggests that aggressive interactions may differ between the two species because of factors not directly related to individual recognition. The decline in aggression within the $P$. metricus trials reported here is similar to the large decline in aggression previously reported in a series of briefer interactions among P. metricus over the course of $4 \mathrm{~d}$ (Sheehan $\&$ Tibbetts 2010). Nevertheless, both species show similar responses to identity information, despite differing in recognition behavior. Therefore, key components of receiver responses to identifiable individuals are shared between the two species, suggesting that similar receiver responses likely existed in their recent common ancestor, prior to the evolution of individual recognition in the $P$. fuscatus lineage.

Individual recognition requires receivers to learn and associate information about individuals with their identity phenotypes (Tibbetts \& Dale 2007; Gherardi et al. 2012). Our data are consistent with learning as a mechanism that leads to reduced aggression toward identifiable wasps in P. metricus. If the responses to our treatment depended on innate preferences or responses, then the color an individual was painted should have a strong influence on how others interacted with them. In contrast, the results of our experiment were not influenced by the particular color an individual was painted (i.e. yellow or black) but rather if they displayed the rare marking within the group. The fact that outcomes are context-dependent is consistent with P. metricus receivers learning to associate information with identifying paint marks and reducing aggression toward individuals with whom they have previously interacted. Additional evidence of learning comes from the observation that at the outset of the trials, levels of aggression directed toward identifiable and indistinguishable individuals are similar but begin to diverge after a few minutes, with identifiable individuals receiving less aggression (Fig. S1). 
Familiarity commonly reduces aggression among a wide range of animals (Jaeger 1981; Godard 1991; Gherardi et al. 2012) and in Polistes wasps in particular (Tibbetts 2002; Sheehan \& Tibbetts 2008). When the relative status between two individuals is unknown, animals commonly engage in aggressive interactions to determine relative status, which may range from brief encounters to escalated bouts (Arnott \& Elwood 2009). Memories of prior interactions allow individuals to assess their relative status and dominance without the need for aggression, leading to reduced aggression and ritualized dominance and subordinance displays (Barnard \& Burk 1979; Van Rhijn \& Vodegel 1980; Tibbetts \& Dale 2007; Carazo et al. 2008). As a result, learning can lead to reduced aggression for identifiable individuals because the outcomes of past interactions can be associated with a particular individual. When individuals are indistinguishable, past interactions with multiple individuals (who may differ in relative dominance status) cannot be differentiated.

Associative learning is required for identity information to reduce aggression. It is not surprising that $P$. metricus are able to associate visual identity information with social interactions. Even in the absence of a history of selection for individual recognition, receiver learning abilities in many species are likely sufficient to mediate discrimination based on identity information. For example, foraging in social insects such as bees and wasps is mediated by associative learning as well as more complex non-elemental forms of learning (Lehrer \& Campan 2004; AvarguesWeber et al. 2011; Dyer 2012). Selection for individual recognition may favor the evolution of more specialized learning mechanisms as has been shown in P. fuscatus (Sheehan \& Tibbetts 2011), although learning and discrimination abilities used in other tasks such as foraging are likely to facilitate discrimination of individuals in our experiment.

The similar receiver responses in $P$. metricus and $P$. fuscatus suggest that the lack of identity information in $P$. metricus senders is a key factor limiting the potential expression of individual recognition in $P$. metricus. On longer evolutionary timescales, however, we do not expect that the lack of identity information presents a significant constraint to the evolution of individual recognition in P. metricus. Color pattern evolution is highly labile in Polistes, and identitysignaling traits are not costly to produce or maintain (Tibbetts 2004; Tibbetts \& Curtis 2007). Therefore, under the appropriate circumstances, existing receiver responses in $P$. metricus should favor the evolution of increased identity information in senders.
If the benefits of identity information in P. metricus are sufficient to select for identity signals, why does $P$. metricus lack variable color patterns? It is likely that identity signals have not evolved in $P$. metricus because there are few social interactions among $P$. metricus foundresses in natural populations, as P. metricus overwhelming initiate solitary nests (Starr 1976; Bohm \& Stockhammer 1977; Hughes et al. 1993; Singer \& Espelie 1996). Interactions among competing co-foundresses are thought to be particularly important for the evolution of social signaling in Polistes (Tibbetts 2004). Cooperating foundresses form a dominance hierarchy in many species that determines relative rates of reproduction and work, with the most dominant foundress monopolizing egg laying (Reeve 1991). Within foundress associations, then, there is the potential for individually differentiated interactions where foundresses invest differently in cooperative and aggressive acts depending on their interaction partners and their relative places in the hierarchy (West Eberhard 1969). Indeed, P. fuscatus, which has highly variable identity signals, frequently founds nests in large foundress associations and forms a strict dominance hierarchy among foundresses (West Eberhard 1969; Reeve et al. 2000).

In contrast to the interactions within foundress associations, the interactions between queens and workers are not thought to favor visual signaling, as there is less reproductive conflict between queens and their daughters (i.e. workers) (Reeve 1991; Tibbetts $\delta$ Sheehan 2013). There is less evidence of individually differentiated interactions among workers within paper wasp colonies, although behavioral profiles among workers in temperate species tend to differ based on their age (Strassmann \& Meyer 1983; Miyano 1986; Hughes \& Strassmann 1988). Additionally, the recognition of queen vs. worker status is communicated using blends of cuticular hydrocarbons that convey information about reproductive state (Monnin 2006). Further, whereas all species of paper wasps have interactions between queens and workers, only those species with foundress associations have variable color patterning associated with visual signaling (Tibbetts 2004). While receiver responses could facilitate the evolution of identity information in P. metricus, there is little opportunity for selection to act given the rarity of interactions among foundresses, which helps explain why $P$. metricus lack identity signals and individual recognition.

The results of this experiment present the possibility that pre-existing receiver behavior could facilitate the evolution of individual recognition. Pre-existing receiver responses have been suggested to favor the 
evolution of a range of sexually selected traits (Endler \& Basolo 1998; Jansson \& Enquist 2005; Kolm et al. 2012), but the role of receiver responses in the evolution of recognition systems has received less attention. Typically, biases in receiver responses manifest as preexisting preferences for particular trait forms [i.e. longer sword tails (Morris et al. 2007), red coloration in sticklebacks (Smith et al. 2004), longer tail plumage in widowbirds (Pryke \& Andersson 2002)]. However, pre-existing receiver behaviors facilitating individual recognition would involve differential responses to unique individuals rather than preferences for particular phenotypes. Receiver responses favorable to identity signaling are likely to be important in the evolution of individual recognition in many taxa. Whether such responses existed prior to selection for recognition or are a result of such selection is an unexplored question. Our data are consistent with preexisting receiver behavior in Polistes, although tests of additional species within the $P$. fuscatus-metricus clade are needed to adequately test such a hypothesis (Fig. 2). Vespid wasps present an excellent opportunity to explore the role of receiver biases as there have been multiple independent evolutions of visual signaling across the group (e.g. individual recognition in Liostenogaster, Baracchi et al. 2012).

At a broader scale, the role of pre-existing receiver behavior in the origin of individual recognition is currently unclear. In many species that lack individual recognition, receivers do not respond to available identity information (McCulloch et al. 1999; Schibler \& Manser 2007; Cure et al. 2009), suggesting that receiver responses do not favor individual recognition in those species. However, it is also possible that the sender phenotypes tested in these experiments appear individually distinctive when analyzed by researchers, but are not actually perceived as individually distinctive by the animals (Tibbetts et al. 2008). Therefore, researchers must consider the perceptual abilities of receivers, as well as the amount of identity information potentially available to receivers when testing the role of receiver responses in the evolution of individual recognition. Receivers may be more likely to respond to variation when such variation is easier to discern.

Individual recognition requires a number of cognitive steps, including distinguishing among individuals, learning individual identity, associating identity with individual specific information and later recalling that information (Tibbetts \& Dale 2007; Gherardi et al. 2012). Although individual recognition appears cognitively complex, relatively little work has examined how cognition differs between closely related species with and without individual recognition, although identifying such differences is crucial to understanding the evolution of complex cognition and behavior (Chittka et al. 2012). In the case of paper wasps, individual recognition is associated with differences in cognitive processing, social memory and color pattern variability (Sheehan \& Tibbetts 2008, 2010, 2011). The present study, however, suggests that previously described differences in individual recognition abilities are a matter of degree rather than kind. The basic cognitive building blocks of individual recognition behavior-receiver discrimination and response-are present and expressed in a species that lacks individual recognition when provided with sufficient identity information. In the case of paper wasps, at least, receiver cognition and behavior does not appear to limit the initial evolution of individual recognition.

\section{Acknowledgements}

We thank Tory Hendry and Carrie Green who helped collect wasps used in this study. We thank Ijams Nature Center and the Columbus MetroParks for permission to collect on their lands. Funding was provided by an Edwin S. George Reserve Scholarship and the University of Michigan.

\section{Literature Cited}

Arnott, G. \& Elwood, R. W. 2009: Assessment of fighting ability in animal contests. Anim. Behav. 77, 991-1004.

Avargues-Weber, A., Deisig, N. \& Giurfa, M. 2011 : Visual cognition in social insects. Annu. Rev. Entomol. 56, 423-443.

Baracchi, D., Petrocelli, I., Cusseau, G., Pizzocaro, L., Teseo, S. \& Turillazzi, S. 2012: Facial markings in the hover wasps: quality signals and familiar recognition cues in two species of Stenogastrinae. Anim. Behav. 85, 203-212.

Barnard, C. J. \& Burk, T. 1979: Dominance hierarchies and the evolution of individual recognition. J. Theor. Biol. 81, 65-73.

Bee, M. A. \& Gerhardt, H. C. 2002: Individual voice recognition in a territorial frog (Rana catesbeiana). Proc. R. Soc. Lond. B Biol. Sci. 269, 1443-1448.

Beecher, M. D. 1988: Kin recognition in birds. Behav. Genet. 18, 465-482.

Beer, C. G. 1969: Laughing gull chicks: recognition of their parent's voices. Science 166, 1030-1032.

Bergman, T. J., Beehner, J. C., Cheney, D. L. \& Seyfarth, R. M. 2003: Hierarchical classification by rank and kinship in baboons. Science 302, 1234-1236.

Bohm, M. K. \& Stockhammer, K. A. 1977: The nesting cycle of a paper wasp, Polistes metricus (Hymenoptera: Vespidae). J. Kansas Entomol. Soc. 50, 275-286. 
Briscoe, A. D. \& Chittka, L. 2001: The evolution of color vision in insects. Annu. Rev. Entomol. 46, 471-510.

Brosnan, S. F., Salwiczek, L. \& Bshary, R. 2010: The interplay of cognition and cooperation. Philos. Trans. R. Soc. B Biol. Sci. 365, 2699-2710.

Bshary, R., Wickler, W. \& Fricke, H. 2002: Fish cognition: a primate's eye view. Anim. Cogn. 5, 1-13.

Buck, M., Cobb, T. P., Stahlhut, J. K. \& Hanner, R. H. 2012: Unravelling cryptic species diversity in eastern Nearctic paper wasps, Polistes (Fuscopolistes), using male genitalia, morphometrics and DNA barcoding, with descriptions of two new species (Hymenoptera: Vespidae). Zootaxa 3502, 1-48.

Carazo, P., Font, E. \& Desfilis, E. 2008: Beyond “nasty neighbours" and "dear enemies"? Individual recognition by scent marks in a lizard (Podarcis hispanica). Anim. Behav. 76, 1953-1963.

Cheney, D. L. \& Seyfarth, R. M. 2007: Baboon Metaphysics: Evolution of a Social Mind. Univ. of Chicago Press, Chicago.

Chittka, L., Rossiter, S. J., Skorupski, P. \& Fernando, C. 2012: What is comparable in comparative cognition? Philos. Trans. R. Soc. B Biol. Sci. 367, 2677-2685.

Crowley, P. H., Provencher, L., Sloane, S., Dugatkin, L. A., Spohn, B., Rogers, L. \& Alfieri, M.1996: Evolving cooperation: the role of individual recognition. Biosystems 37, 49-66.

Cure, C., Aubin, T. \& Mathevon, N. 2009: Acoustic convergence and divergence in two sympatric burrowing nocturnal seabirds. Biol. J. Linn. Soc. 96, 115-134.

Dale, J., Lank, D. B. \& Reeve, H. K. 2001: Signaling individual identity versus quality: a model and case studies with ruffs, queleas, and house finches. Am. Nat. 158, $75-86$.

Doorn, G. S. V., Hengeveld, G. M. \& Weissing, F. J. 2003:

The evolution of social dominance II: multi-player models. Behaviour 140, 1333-1358.

Dreier, S. \& D'Ettorre, P. 2009: Social context predicts recognition systems in ant queens. J. Evol. Biol. 22, 644-649.

Dunbar, R. I. M. \& Shultz, S. 2007: Evolution in the social brain. Science 317, 1344-1347.

Dyer, A. G. 2012: The mysterious cognitive abilities of bees: why models of visual processing need to consider experience and individual differences in animal performance. J. Exp. Biol. 215, 387-395.

Dyer, A. G., Neumeyer, C. \& Chittka, L. 2005: Honeybee (Apis mellifera) vision can discriminate between and recognise images of human faces. J. Exp. Biol. 208, 4709-4714.

Endler, J. A. \& Basolo, A. L. 1998: Sensory ecology, receiver biases and sexual selection. Trends Ecol. Evol. 13, 415-420.

Gherardi, F. \& Atema, J. 2005: Memory of social partners in hermit crab dominance. Ethology 111, 271-285.
Gherardi, F., Aquiloni, L. \& Tricarico, E. 2012: Revisiting social recognition systems in invertebrates. Anim. Cogn. 15, 745-762.

Godard, R. 1991: Long-term-memory of individual neighbors in a migratory songbird. Nature 350, 228-229.

Hirose, Y. \& Yamasaki, M. 1984: Dispersal of females for colony founding in Polistes jadwigae Dalla Torre (Hymenoptera, Vespidae). Kontyu 52, 65-71.

Hughes, C. R. \& Strassmann, J. E. 1988: Age is more important than size in determining dominance among workers in the primitively eusocial wasp, Polistes instabilis. Behaviour 107, 1-14.

Hughes, C. R., Queller, D. C., Strassmann, J. E. \& Davis, S. K. 1993: Relatedness and altruism in Polistes Wasps. Behav. Ecol. 4, 128-137.

Jaeger, R. G. 1981: Dear enemy recognition and the costs of aggression between salamanders. Am. Nat. 117, 962 -974 .

Jansson, L. \& Enquist, M. 2005: Testing the receiver bias hypothesis empirically with "virtual evolution". Anim. Behav. 70, 865-875.

Johnstone, R. A. 1997: Recognition and the evolution of distinctive signatures: when does it pay to reveal identity? Proc. R. Soc. Lond. B Biol. Sci. 264, 1547-1553.

Klahn, J. E. 1979: Philopatric and non-philopatric foundress associations in the social wasp Polistes fuscatus. Behav. Ecol. Sociobiol. 5, 417-424.

Kolm, N., Amcoff, M., Mann, R. P. \& Arnqvist, G. 2012: Diversification of a food-mimicking male ornament via sensory drive. Curr. Biol. 22, 1440-1443.

Lehmann, L. \& Perrin, N. 2002: Altruism, dispersal, and phenotype-matching kin recognition. Am. Nat. 159, 451-468.

Lehrer, M. \& Campan, R. 2004: Shape discrimination by wasps (Paravespula germanica) at the food source: generalization among various types of contrast. J. Comp. Physiol. A Neuroethol. Sens. Neural Behav. Physiol. 190, 651-663.

Liebert, A. E. \& Starks, P. T. 2004: The action component of recognition systems: a focus on the response. Ann. Zool. Fenn. 41, 747-764.

Loesche, P., Stoddard, P. K., Higgins, B. J. \& Beecher, M. D. 1991: Signature versus perceptual adaptations for individual vocal recognition in swallows. Behaviour 118, 15-25.

Makino, S., Yamane, S., Sunose, T. \& Aoki, S. 1987: Dispersion distance of queens from natal sites in the two haplometrotic paper wasps Polistes riparius and P. snelleni (Hymenoptera: Vespidae). Res. Popul. Ecol. 29, $111-117$.

McCulloch, S., Pomeroy, P. P. \& Slater, P. J. B. 1999: Individually distinctive pup vocalizations fail to prevent allo-suckling in grey seals. Can. J. Zool. 77, 716-723.

Miyano, S. 1986: Colony development, worker behavior and male production in orphan colonies of a Japanese 
paper wasp, Polistes chinensis antennalis Pérez (Hymenoptera: Vespidae). Res. Popul. Ecol. 28, 347-361.

Monnin, T. 2006: Chemical recognition of reproductive status in social insects. Ann. Zool. Fenn. 43, 515530.

Morris, M. R., Tudor, M. S. \& Dubois, N. S. 2007: Sexually selected signal attracted females before deterring aggression in rival males. Anim. Behav. 74, 1189-1197.

Pickett, K. M. \& Carpenter, J. M. 2010: Simultaneous analysis and the origin of Eusociality in the Vespidae (Insecta: Hymenoptera). Arthropod Syst. Phylogeny 68, 3 -33.

Pryke, S. R. \& Andersson, S. 2002: A generalized female bias for long tails in a short-tailed widowbird. Proc. R. Soc. B Biol. Sci. 269, 2141-2146.

Reeve, H. K. 1991: Polistes. In: The Social Biology of Wasps (Ross, K. G. \& Matthews, R. W., eds). Cornell Univ. Press, Ithaca, NY, pp. 99-149.

Reeve, H. K. \& Nonacs, P. 1992: Social contracts in wasp societies. Nature 359, 823-825.

Reeve, H. K., Starks, P. T., Peters, J. M. \& Nonacs, P. 2000: Genetic support for the evolutionary theory of reproductive transactions in social wasps. Proc. R. Soc. Lond. B Biol. Sci. 267, 75-79.

Roeseler, P. F. 1991. Reproductive competition during colony establishment. In: The Social Biology of Wasps (Ross, K. G. \& Matthews, R. W., eds). Cornell Univ. Press, Ithaca, NY, pp. 309-335.

Schibler, F. \& Manser, M. B. 2007: The irrelevance of individual discrimination in meerkat alarm calls. Anim. Behav. 74, 1259-1268.

Scott-Phillips, T. C., Blythe, R. A., Gardner, A. \& West, S. A. 2012: How do communication systems emerge? Proc. R. Soc. B Biol. Sci. 279, 1943-1949.

Sheehan, M. J. \& Tibbetts, E. A. 2008: Robust long-term social memories in a paper wasp. Curr. Biol. 18, R851-R852.

Sheehan, M. J. \& Tibbetts, E. A. 2009: Evolution of identity signals: frequency-dependent benefits of distinctive phenotypes used for individual recognition. Evolution 63, 3106-3113.

Sheehan, M. J. \& Tibbetts, E. A. 2010: Selection for individual recognition and the evolution of polymorphic identity signals in Polistes paper wasps. J. Evol. Biol. 23, $570-577$.

Sheehan, M. J. \& Tibbetts, E. A. 2011: Specialized face learning is associated with individual recognition in paper wasps. Science 334, 1272-1275.

Sherman, P. W., Reeve, H. K., Pfennig, D. W., Krebs, J. R. E Davies, N. B. 1997: Recognition systems. In: Behavioural Ecology: An Evolutionary Approach (Krebs, J. \& Davies, N., eds). Blackwell Science Ltd., Oxford, pp. 69-96.
Singer, T. L. \& Espelie, K. E. 1996: Nest surface hydrocarbons facilitate nestmate recognition for the social wasp, Polistes metricus say (Hymenoptera: Vespidae). J. Insect Behav. 9, 857-870.

Smith, C., Barber, I., Wootton, R. J. \& Chittka, L. 2004: A receiver bias in the origin of three-spined stickleback mate choice. Proc. R. Soc. Lond. B Biol. Sci. 271, 949-955.

Sokal, R. R. \& Rohlf, F. J. 1995: Biometry. New York: W.H. Freeman and Company.

Stamps, J. A. 1987: The effect of familiarity with a neighborhood on territory acquisition. Behav. Ecol. Sociobiol. 21, 273-277.

Starr, C. K. 1976: Nest reutilization by Polistes metricus (Hymenoptera: Vespidae) and possible limitation of multiple foundress associations by parasitoids. J. Kansas Entomol. Soc. 49, 142-144.

Stevens, J. R., Cushman, F. A. \& Hauser, M. D. 2005: Evolving the psychological mechanisms for cooperation. Annu. Rev. Ecol. Evol. Syst. 36, 499-518.

Strassmann, J. E. \& Meyer, D. C. 1983: Gerontocracy in the social wasp, Polistes exclamans. Anim. Behav. 31, $431-438$.

Strassmann, J. E., Fortunato, A., Cervo, R., Turillazzi, S., Damon, J. M. \& Queller, D. C. 2004: The cost of queen loss in the social wasp Polistes dominulus (Hymenoptera: Vespidae). J. Kansas Entomol. Soc. 77, 343-355.

Tibbetts, E. A. 2002: Visual signals of individual identity in the wasp Polistes fuscatus. Proc. R. Soc. Lond. B Biol. Sci. 269, 1423-1428.

Tibbetts, E. A. 2004: Complex social behaviour can select for variability in visual features: a case study in Polistes wasps. Proc. R. Soc. Lond. B Biol. Sci. 271, 19551960.

Tibbetts, E. A. \& Curtis, T. R. 2007: Rearing conditions influence quality signals but not individual identity signals in Polistes wasps. Behav. Ecol. 18, 602-607.

Tibbetts, E. A. \& Dale, J. 2007: Individual recognition: it is good to be different. Trends Ecol. Evol. 22, 529-537.

Tibbetts, E. A. \& Sheehan, M. J. 2013: Individual recognition and the evolution of learning and memory in Polistes paper wasps. In: Invertebrate Learning and Memory (Menzel, R. \& Benjamin, P., eds). Elsevier, San Diego, CA, pp. 561-572.

Tibbetts, E. A., Sheehan, M. J. \& Dale, J. 2008: A testable definition of individual recognition. Trends Ecol. Evol. 23, 356-356.

Torriani, M. V. G., Vannoni, E. \& McElligott, A. G. 2006: Mother-young recognition in an ungulate hider species: a unidirectional process. Am. Nat. 168, 412-420.

Van Rhijn, J. G. \& Vodegel, R. 1980: Being honest about one's intentions: an evolutionary stable strategy for animal conflicts. J. Theor. Biol. 85, 623-641. 
West Eberhard, M. J. 1969: The Social Biology of Polistine Wasps. Museum of Zoology, Univ. of Michigan, Ann Arbor.

Wilson, E. 1975: Sociobiology: The New Synthesis. Harvard, Cambridge.

\section{Supporting Information}

Additional supporting information may be found in the online version of this article:

Figure. S1: Patterns of aggression over time. 